\title{
SOME REMARKS ON FOLIATIONS WITH MINIMAL LEAVES
}

\author{
ANDRÉ HAEFLIGER
}

Let $\mathcal{F}$ be a foliation on a manifold $X$ of dimension $n=p+q$, the leaves being submanifolds of dimension $p$ and codimension $q$. Everything will be assumed to be of class $C^{\infty}$. The question of the existence of a riemannian metric on $X$ for which all the leaves are minimal submanifolds has been discussed by Rummler [5] and Sullivan [7]. Assume for simplicity that the tangent bundle $T \mathscr{F}$ of the leaves of the foliation is orientable and oriented. They prove the following criterion.

Theorem (Rummler-Sullivan.) Let $g_{0}$ be a smooth scalar product on $T \mathcal{F}$. It is induced by a riemannian metric $g$ on $X$ for which the leaves are minimal submanifolds iff the volume $p$-form $\omega_{0}$ on the leaves defined by $g_{0}$ (and the orientation) is the restriction to the leaves of a p-form $\omega$ on $X$ which is relatively closed, namely, $d \omega\left(\xi_{1}, \cdots, \xi_{p+1}\right)=0$ if the first $p$ vector fields $\xi_{i}$ are tangent to the leaves.

Using the above criterion Rummler and Sullivan proved the existence or the nonexistence of such a metric in many interesting cases. Our goal is to prove that for a compact $X$ the above condition depends only on the transverse structure of $\mathscr{F}$, and to deduce from this some consequences.

We first give a short proof of the Rummler-Sullivan criterion. Let $\nu$ be a vector field on a small open set $U$ of $X$ such that the local flow $\varphi_{t}$ generated by $\nu$ maps leaves to leaves. Let $K_{0} \subset U$ be a piece of a leaf and let $K_{t}=\varphi_{t}\left(K_{0}\right)$. Consider a $p$-form $\omega$ on $X$ extending $\omega_{0}$. Then for $t=0$

$$
\frac{d}{d t}\left(\text { volume } K_{t}\right)=\frac{d}{d t} \int_{K_{t}} \omega=\frac{d}{d t} \int_{K_{0}} \varphi_{t}^{*} \omega=\int_{K_{0}} \nu \cdot \omega=\int_{K_{0}} d i_{\nu} \omega+i_{\nu} d \omega
$$

where $\nu . \omega$ is the Lie derivative of $\omega$ in the direction of $\nu$. Assume there is a metric $g$ extending $g_{0}$ such that all the leaves are minimal. Define $\omega$ such that $i_{\nu} \omega$ vanishes on each leaf for $\nu$ orthogonal to the leaves. For such a $\nu$, $(d / d t)$ (volume $\left.K_{t}\right)=0$, hence the above formula shows that $d i_{\nu} \omega$ vanishes on the leaves, so $\omega$ is relatively closed.

Received March 20, 1979, and, in revised form, November 24, 1979. 
Conversely, let $\omega$ be a relatively closed form extending $\omega_{0}$. At a point $x \in X$, the vectors $\xi$ such that $i_{\xi} \omega=0$ form a vector subspace $N_{x}$ of $T_{x} X$ complementary to the tangent space of the leaf through $x$. Consider any metric $g$ extending $g_{0}$ and such that $N_{x}$ is orthogonal to the tangent space of the leaf at $x$, for all $x \in X$. Then the above formula shows that for $\nu$ orthogonal to the leaves, the first variatiion $(d / d t)$ (volume $\left.K_{t}\right)$ is zero for any piece $K_{0}$ in a leaf. So each leaf is a minimal submanifold.

\section{FORMS AND CURRENTS ON THE TRANSVERSE STRUCTURE OF A FOLIATION}

\subsection{Morphisms of pseudogroups}

Recall that a pseudogroup $H$ of diffeomorphisms of a manifold $T$ is a collection of diffeomorphisms of open sets of $T$ on open sets of $T$, which contains the identity map of $T$ and is closed under composition (whenever it is defined), inverses, restrictions to open sets, and unions.

Consider two pseudogroups $H$ and $H^{\prime}$ of diffeomorphisms of $T$ and $T^{\prime}$ respectively. A morphism $\Phi: H \rightarrow H^{\prime}$ is a collection $\Phi$ of diffeomorphism of open sets of $T$ on open sets of $T^{\prime}$ such that:

(i) the sources of the $\varphi \in \Phi$ cover $T$,

(ii) if $h \in H$ and $\varphi_{1}, \varphi_{2} \in \Phi$, then $\varphi_{1} h \varphi_{2}^{-1} \in H^{\prime}$,

(iii) if $h \in H, h^{\prime} \in H^{\prime}, \varphi \in \Phi$, then $h^{\prime} \varphi h \in \Phi$,

(iv) $\Phi$ is closed under unions.

Any collection $\Phi_{0}$ such that

(a) the $H$-orbit of each point of $T$ intersects the source of a $\varphi \in \Phi$,

(b) if $h \in H$, and $\varphi_{1}, \varphi_{2} \in \Phi_{0}$, then $\varphi_{1} h \varphi_{2}^{-1} \in H^{\prime}$ can be uniquely completed as a collection $\Phi$ satisfying (i)-(iv) by considering all unions of elements of the form $h^{\prime} \varphi h, \varphi \in \Phi, h \in H, h^{\prime} \in H^{\prime}$. Such a $\Phi_{0}$ will be called an atlas generating the morphism $\Phi$.

If $\Phi^{\prime}$ is a morphism of $H^{\prime}$ in $H^{\prime \prime}$, then the collection of all $\varphi^{\prime} . \varphi, \varphi \in \Phi$, $\varphi \in \Phi^{\prime}$, generates a morphism of $H$ in $H^{\prime \prime}$. Under this composition, morphisms form a category. $\Phi_{0}$ generates an isomorphism (or an equivalence) of $H$ on $H^{\prime}$ iff the union of the targets of the $\varphi \in \Phi_{0}$ intersects each orbit of $H^{\prime}$ and $\varphi_{2}^{-1} h^{\prime} \varphi_{1} \in H$ for any $\varphi_{1}, \varphi_{2} \in \Phi_{0}, h^{\prime} \in H$. In that case we say that $H$ is equivalent to $H^{\prime}$. For instance, let $U$ be an open subset of $T$ and let $H_{U}$ be the pseudogroup of diffeomorphisms of $U$ whose elements are the restriction to $U$ of the elements of $H$. Then the inclusion of $U$ in $T$ generates a morphism of $H_{U}$ in $H$, and is an isomorphism iff $U$ meets each orbit of $H$. In the case where the space $T / H$ of $H$-orbits is a differentiable manifold, the 
natural projection $p: T \rightarrow T / H$ being locally a diffeomorphism, $H$ is equivalent to the trivial pseudogroup on $T / H$ (generated by the identity).

\subsection{Forms and currents on $T / H$}

Let $\Omega_{c}^{p}(T)$ be the vector space of smooth $p$-forms on $T$ with compact support, and denote by $\Omega_{c}^{p}(T / H)$ the quotient of $\Omega_{c}^{p}(T)$ by the vector subspace generated by elements of the form $\alpha-h^{*} \alpha$, where $h \in H$, and $\alpha$ is a $p$-form with compact support in the range of $h$. On $\Omega_{c}^{p}(T / H)$ we consider the topology obtained by taking the quotient of the usual $C^{\infty}$-topology on $\Omega_{c}^{p}(T)$. In general this topology is not Hausdorff (see examples below).

The exterior differential $d: \Omega_{c}^{p}(T) \rightarrow \Omega_{c}^{p+1}(T)$ induces a continuous differential

$$
d: \Omega_{c}^{p}(T / H) \rightarrow \Omega_{c}^{p+1}(T / H) .
$$

Thus we associated to $H$ a differential graded topological vector space $\Omega_{c}^{*}(T / H)$. We shall see below that it depends only on the equivalence class of $H$, and its dual is naturally isomorphic to the space of invariant currents on $T$. Indeed let $C_{p}(T)$ be the space of $p$-currents on $T$, namely, the vector space of continuous linear forms on $\Omega_{c}^{p}(T)$. A p-current $c$ is invariant by $H$ if for any $h \in H$ and any $p$-form $\alpha$ with support in the range of $h$, then $c(\alpha)=$ $c\left(h^{*} \alpha\right)$. So it defines a continuous linear form on the quotient $\Omega_{c}^{p}(T / H)$. If $\alpha \in \Omega_{c}^{p}(T)$ is such that $c(\alpha)=0$ for all invariant current $c$, the class of $\alpha$ in $\Omega_{c}^{p}(T / H)$ is not zero in general, but is in the closure of the kernel of the projection $\Omega_{c}^{p}(T) \rightarrow \Omega_{c}^{p}(T / H)$. For this reason, it is in general easier to describe the space $C^{p}(T)^{H}$ of invariant currents.

Proposition. A morphism $\Phi$ of $H$ in $H^{\prime}$ induces functorially a continuous morphism of differential graded vector spaces

$$
\Phi^{*}: \Omega_{c}^{*}(T / H) \rightarrow \Omega_{c}^{*}\left(T^{\prime} / H^{\prime}\right) .
$$

Proof. We can express each $\alpha \in \Omega_{c}^{p}(T)$ as a finite sum

$$
\alpha=\sum_{\varphi \in \Phi} \alpha_{\varphi}
$$

where $\alpha_{\varphi}$ is a $p$-form with compact support in the source $U_{\varphi}$ of $\varphi \in \Phi$, and is zero except for a finite number of $\phi$.

The map $\Phi^{*}$ associates to the class of $\alpha$ the class $\Sigma_{\varphi}\left(\varphi^{-1}\right)^{*} \alpha_{\varphi}$ in $\Omega_{c}^{p}\left(T^{\prime} / H^{\prime}\right)$.

We have to check that this definition is independent of the choice of the decomposition of $\alpha$ and the choice of $\alpha$ in its class. For the first part, it is sufficient to note that if $\lambda_{\varphi}$ is a partition of unity subordinated to the covering 
$U_{\varphi}$ of $T$, then

$$
\sum_{\varphi}\left(\varphi^{-1}\right)^{*} \alpha_{\varphi}=\sum_{\varphi, \psi \in \Phi}\left(\varphi^{-1}\right)^{*} \lambda_{\psi} \alpha_{\varphi}
$$

is equivalent to

$$
\sum_{\varphi, \psi}\left(\varphi \psi^{-1}\right)^{*}\left(\varphi^{-1}\right)^{*} \lambda_{\psi} \alpha_{\varphi}=\sum_{\varphi, \psi}\left(\psi^{-1}\right)^{*} \lambda_{\psi} \alpha_{\varphi}=\sum_{\psi}\left(\psi^{-1}\right)^{*} \lambda_{\psi} \alpha
$$

because $\varphi \psi^{-1} \in H^{\prime}$.

Assume now that $\alpha=\beta-h^{*} \beta$, where $\beta$ has its support in the range of $h \in H$. We can express $\beta$ as a finite sum $\sum \beta_{\varphi}$, where the support of $\beta_{\varphi}$ is in the source of $\varphi$, and the support of $h^{*} \beta_{\varphi}$ is in the source of some $\psi \in \Phi$ (for this, it is sufficient to multiply $\beta$ by a partition of unity subordinated to the covering of the range of $h$ by the intersections $\left.U_{\varphi} \cap h\left(U_{\psi}\right), \varphi, \psi \in \Phi\right)$. Then $\alpha=\Sigma_{\varphi} \beta_{\varphi}-h^{*} \beta_{\varphi}$ can be mapped on

$$
\sum\left(\varphi^{-1}\right)^{*} \beta_{\varphi}-\sum\left(\psi^{-1}\right)^{*} h^{*} \beta_{\varphi}=\sum\left(\varphi^{-1}\right)^{*} \beta_{\varphi}-\sum\left(\psi^{-1}\right)^{*} h^{*} \varphi^{*}\left(\varphi^{-1}\right)^{*} \beta_{\varphi},
$$

which is equivalent to zero because $\varphi h \psi^{-1} \in H^{\prime}$.

It is straightforward to check that $\Phi^{*}$ commutes with $d$ and is continuous.

Corollary. An isomorphism of $H$ on $H^{\prime}$ induces a topological isomorphism of $\Omega_{c}^{*}(T / H)$ on $\Omega_{c}^{*}\left(T^{\prime} / H^{\prime}\right)$.

In particular, if $H$ acts on $T$ in a properly discontinuous way, i.e., if the map $T \rightarrow T / H$ is locally a diffeomorphism, then $\Omega_{c}^{p}(T / H)$ is just isomorphic to the vector space of $p$-forms with compact support on $T / H$.

Also if each point $x$ of $T$ has a neighborhood $V$ such that the restriction of $H$ to $V$ is generated by a finite group of diffeomorphisms of $V$, then $T / H$ is a manifold in the sense of Satake, and $\Omega_{c}^{p}(T)$ is what is usually called the space of differential forms on $T / H$.

\subsection{The holonomy pseudogroup of a foliation}

Let $\mathcal{F}$ be a foliation of codimension $q$ on a manifold $X$. A transversal submanifold $T$ is a manifold of dimension $q$ together with an immersion $t: T \rightarrow X$ which is transversal to the leaves.

Given two points $x_{1}, x_{2}$ in $T$ such that $t\left(x_{1}\right)$ and $t\left(x_{2}\right)$ are in the same leaf $L$, then a homotopy class of paths $\gamma$ joining $t\left(x_{1}\right)$ to $t\left(x_{2}\right)$ in $L$ determines a germ at $x_{1}$ of a diffeomorphism $h$ of a neighborhood of $x_{1}$ on a neighborhood of $x_{2}$, called the holonomy defined by the path $\gamma$ : if $x$ is close to $x_{1}$, there is a path close to $\gamma$ and contained in a leaf, joining $t(x)$ to $t(h x)$. The holonomy pseudogroup induced by $\mathscr{F}$ on $T$ is the pseudogroup whose elements are local diffeomorphisms of $T$ whose germs at each point are determined in this way. 
The transversal submanifold $t: T \rightarrow X$ will be said to be complete if $t(T)$ cuts every leaf of $\mathscr{F}$. If $t^{\prime}: T^{\prime} \rightarrow X$ is another complete transversal submanifold, then the holonomy pseudogroup $H^{\prime}$ induced on $T^{\prime}$ is canonically equivalent to $H$. Indeed the set $\Phi$ of elements of the holonomy pseudogroup induced on the disjoint union of $T$ and $T^{\prime}$ with source in $T$ and range in $T^{\prime}$ is a morphism of $H$ in $H^{\prime}$. Hence to each foliation $\mathcal{F}$ we can associate a well defined equivalence class of pseudogroups, namely, the class of any holonomy pseudogroup $H$ induced by $\mathscr{F}$ on a complete transversal submanifold $T$. By abuse of language, such an $H$ will be called the (transverse) holonomy pseudogroup of $\mathcal{F}$.

Definition. We shall denote by $\Omega_{c}^{*}(\operatorname{Tr} \mathscr{F})$ the topological differential graded vector space of forms on $T / H$, where $H$ is the holonomy pseudogroup induced on a complete transversal submanifold $T$. This definition is independent of the choice of the transversal $T$, because if $H^{\prime}$ is the holonomy pseudogroup induced on a complete transversal submanifold $T^{\prime}$, then $\Omega_{c}^{*}(T / H)$ is canonically isomorphic to $\Omega_{c}^{*}\left(T^{\prime} / H^{\prime}\right)$.

A continuous linear form on $\Omega_{c}^{k}(\mathrm{Tc} \mathscr{F})$ will be called an holonomy invariant $k$-current. In other words, it is a $k$-current defined on every transversal submanifold, and is invariant by holonomy. The vector space of invariant $k$-currents will be denoted by $C_{k}(\operatorname{Tr} \mathscr{F})$. This is the natural generalization of the concept of holonomy invariant measure (cf. [2]). An invariant o-current will also be called an invariant distribution.

Let $f: X^{\prime} \rightarrow X$ be a differentiable map transverse to $\mathscr{F}$, and let $\mathscr{F}^{\prime}=$ $f^{-1}(\mathscr{F})$ be the foliation on $X^{\prime}$ inverse image by $f$ of $\mathscr{F}$. An immersion $t: T \rightarrow X^{\prime}$ is a transversal submanifold to $\mathscr{F}^{\prime}$ iff $f \circ t$ is a transversal submanifold to $\mathscr{F}$. One has a well-defined morphism of the holonomy pseudogroup induced by $\mathscr{F}^{\prime}$ on $T$ in the holonomy pseudogroup induced by $\mathscr{F}$ on $f \circ T$, hence a functorial morphism

$$
\Omega_{c}^{*}\left(\operatorname{Tr} f^{-1} \mathscr{F}\right) \rightarrow \Omega_{c}^{*}(\operatorname{Tr} \mathscr{F}) .
$$

A regular covering of $\mathscr{F}$ will be a covering of $X$ by open sets $U_{i}$ such that:

(i) The space of leaves of the foliation $\mathscr{F}_{i}$ induced by $\mathscr{F}_{F}$ on $U_{i}$ is a $q$-manifold $T_{i}$, the natural projection $f_{i}: U_{i} \rightarrow T_{i}$ being a submersion. The inverse images $f^{-1}(y), y \in T_{i}$, are the plaques in $U_{i}$.

(ii) Each plaque $f_{i}^{-1}\left(y_{i}\right)$ in $U_{i}$ meets at most one plaque $f_{y}^{-1}\left(y_{j}\right)$.

Let $h_{j i}$ be the diffeomorphism mapping $y_{i}$ on $y_{j}$; it is a diffeomorphism of an open set of $T_{i}$ on an open set of $T_{j}$. Let $T$ be the disjoint union of the $T_{i}$, and let $H$ be the pseudogroup generated by the $h_{i j}$. It is easy to see that it is equivalent to the holonomy pseudogroup of $\mathscr{F}$; it will be called the holonomy pseudogroup associated to the regular covering $\left\{U_{i}\right\}$. 


\section{EXAMPLES}

\subsection{Foliations given by closed 1 -forms}

Let $T$ be the circle $R / Z$, and let $H$ be the pseudogroup generated by a rotation $x \mapsto x+\rho$, where $\rho$ is an irrational number. The Lebesgue measure is invariant by $H$, and any invariant distribution (or $o$-current) is a multiple of this measure. Any invariant 1-current is a multiple of the current defined by integration on $H$. Hence $C_{0}(T)^{H}$ and $C_{1}(T)^{H}$ are 1-dimensional.

Suppose that $\rho$ satisfies a diophantine condition: namely, there are positive numbers $s$ and $c$ such that

$$
|m \rho+n| \geqslant \frac{c}{\left(1+m^{2}\right)^{s}},
$$

for any integers $m, n \neq(0,0)$. Then $\Omega^{0}(T / H)$ and $\Omega^{1}(T / H)$ are isomorphic to $R$. Otherwise, $\rho$ is called a Liouville number; then $\Omega^{0}(T / H)$ and $\Omega^{1}(T / H)$ are not Hausdorff, but their quotient by the closure of 0 is still isomorphic to $R$.

The proof of these facts is a standard argument using Fourier series expansion. A function $f$ on $T$ with Fourier series $\Sigma_{m} f_{m} e^{2 i \pi m x}$ is $C^{\infty}$ iff for each positive integer $k$, there is a constant $c$ such that

$$
\left|f_{m}\right|<\frac{c}{\left(1+m^{2}\right)^{k}} .
$$

$f$ is 0 in $\Omega^{0}(T / H)$ iff there is a $C^{\infty}$-function $g$ such that $f(x)=g(x)-g(x+$ $\rho)$. A necessary condition is $\int_{T} f(x) d x=f_{0}=0$, and the Fourier coefficients $g_{m}, m \neq 0$, are uniquely defined ( $\rho$ is irrational). If $\rho$ satisfies a diophantine condition, $g_{m}$ will be the Fourier coefficients of a $C^{\infty}$-function $g$; if $\rho$ is a Liouville number, this will not be the case for a general $f$.

Let $\mathcal{F}$ be a foliation given on a compact manifold by a closed 1-form $\omega$; the cohomology class of $\omega$ defines a homomorphism of $H_{1}(X, Z)$ in $R$ whose image is called the group $P$ of periods of $\omega$. The holonomy pseudogroup is equivalent to the pseudogroup of $T$ generated by the rotations $x \rightarrow x+$ $\alpha / \alpha_{0}$, where $\alpha_{0}$ is a fixed nonzero period and $\alpha \in P$. The rank of $P$ is at least one and is larger than one iff every leaf is dense.

More generally, suppose that $\mathscr{F}$ is given by $q$ independent closed 1-forms. They define a homomorphism of $H_{1}(X, Z)$ in $\mathbf{R}^{q}$ whose image $P$ (the group of periods) is of rank $q$ over $\mathbf{R}$. If $X$ is compact, the holonomy pseudogroup is equivalent to the pseudogroup of transformations of $\mathbf{R}^{q}$ generated by the translations belonging to $P$. For everywhere-dense leaves, this is equivalent to the existence of periods $\alpha, \beta_{1}, \cdots, \beta_{q} \in P$ such that $\beta_{1}, \cdots, \beta_{q}$ are linearly independent over $\mathbf{R}$, and $\alpha=a_{1} \beta_{1}+\cdots+a_{q} \beta_{q}$, where the real numbers 
$1, a_{1}, \cdots, a_{q}$ are linearly independent over the rationals $Q$. The space $C_{k}(\operatorname{Tr} \mathscr{F})$ of $k$-invariant currents is isomorphic to the space of $(q-k)$-forms on $R^{q}$ invariant by all translations. The quotient of $\Omega^{k}(\operatorname{Tr} \mathscr{F})$ by the closure of 0 is isomorphic to the invariant $k$-forms on $\mathbf{R}^{q}$, namely, to the dual of the $k$-exterior power $\Lambda^{k} \mathbf{R}^{q}$ of $\mathbf{R}^{q}$. However, if $\left(a_{1}, \cdots, a_{q}\right)$ satisfies a diophantine condition (cf. for instance Hermann [1]), then $\Omega^{k}(\operatorname{Tr} \mathscr{F}$ ) is actually isomorphic to the dual of $\Lambda^{k} R^{q}$.

The previous examples are particular cases of transversely homogeneous foliations. Let $G / H$ be a homogeneous space, where $H$ is a closed subgroup of the Lie group $G$. We assume that $G$ acts effectively on $G / H$ and that $G / H$ is simply connected. A transversely homogeneous foliation $\mathscr{F}$ on $X$ is given by an open covering $\left\{U_{i}\right\}$ and local submersions $f_{i}: U_{i} \rightarrow G / H$ such that the transition diffeomorphisms $h_{i j}$ are restrictions of translations of $G / H$ by elements of $G$. To such a foliation is associated a homomorphism

$$
\Phi: \pi_{1}(X, x) \rightarrow G
$$

whose image $\Gamma$ is called the global holonomy group of $\mathscr{F}$. On the covering $\tilde{X}$ of $X$ corresponding to the kernel of $\Phi$, the induced foliation $\tilde{\mathscr{F}}$ is given by a submersion $f: \tilde{X} \rightarrow G / H$ which is $\Gamma$-equivariant, $\Gamma$ acting on $X$ by covering translations (cf. Haefliger, Comment. Math. Helv. 32 (1958) 280-281).

If $X$ and $H$ are compact, it is easy to see that $f$ is a fiber map with connected fibers. Then it follows that the holonomy pseudogroup of $\mathcal{F}$ is generated by $\Gamma$ acting on $G / H$.

In general, for an homogeneous space $G / H$ of dimension $n$ (for which the action of $G$ preserves an orientation), the $k$-currents invariant by $G$ are given by the $G$-invariant ( $n-k$ )-forms on $G / H$ (such a form $\alpha$ defines the current $c$ associating to a $k$-form $\omega$ with compact support on $G / H$ the number $\left.\int \alpha \wedge \omega\right)$.

If $X$ and $H$ are compact, $\mathscr{F}$ has an everywhere-dense leaf iff $\Gamma$ is dense in $G$. In that case the holonomy invariant currents are precisely the $G$-invariant forms on $G / H$.

\subsection{Reeb component}

Let $R$ be the solid torus $S^{1} \times D^{2}$ with a Reeb foliation such that the infinitesimal holonomy group of $\partial R$ is nontrivial. Then the holonomy pseudogroup is equivalent to the pseudogroup $H$ of transformations of $T=[0, \infty[$ generated by $h: x \rightarrow \lambda x$, where $0<\lambda<1$. Thus $\Omega_{c}^{0}(T / H)$ is isomorphic to the space of $h$-invariant $C^{\infty}$ functions on ]0, $\infty$ [ (which is itself isomorphic to space of $C^{\infty}$ functions on the circle). The isomorphism maps the class of 
$f \in \Omega_{c}^{0}(T)$ on the function on $] 0, \infty[$ given by

$$
x \rightarrow \sum_{m=-\infty}^{+\infty} \lambda^{m} x f^{\prime}\left(\lambda^{m} x\right)
$$

Similarly $\Omega_{c}^{1}(T / H)$ is isomorphic to the space of $h$-invariant 1 -forms on ] $0, \infty[$.

\section{INTEGRATION ALONG THE LEAVES}

3.1. Theorem. $\mathcal{F}$ be a foliation on $X$ with leaves of dimension $p$, and assume that the tangent bundle to the leaves is oriented. Then there is a continuous open surjective linear map

$$
\int_{\mathscr{F}}: \Omega_{c}^{p+k}(X) \rightarrow \Omega_{c}^{k}(\operatorname{Tr} \mathscr{F})
$$

which commutes with $d$.

Proof. The construction is directly inspired by the construction of the Ruelle-Sullivan current associated to an invariant measure [4].

First recall that if $f: X \rightarrow Y$ is a submersion of a $(p+q)$-manifold $X$ in a $q$-manifold $Y$, the fibers $f^{-1}(y)$ being coherently oriented, there is a continuous map

$$
\int_{\mathscr{F}}: \Omega_{c}^{p+k}(X) \rightarrow \Omega_{c}^{k}(Y)
$$

commuting with $d$. If $\omega$ has its support in a coordinate neighborhood where $f$ is expressed as the linear projection

$$
\begin{aligned}
& f\left(x^{\prime}, \cdots, x^{p}, y^{\prime}, \cdots, y^{q}\right)=\left(y^{\prime}, \cdots, y^{q}\right) \\
& \qquad \begin{aligned}
\omega & =\sum_{J} a_{J}(x, y) d y^{J} \wedge d x^{i} \wedge \cdots \wedge d x^{p} \\
& + \text { terms of degree }<p \text { in the } x^{i},
\end{aligned}
\end{aligned}
$$

then

$$
\int_{f} \omega=\left(\sum \int a_{J}(x, y) d x^{1} \cdots d x^{p}\right) d y^{J} .
$$

Let $\left\{U_{i}\right\}$ be a regular covering of $X$ for $\mathscr{F}$, with projections $f_{i}: U_{i} \rightarrow T_{i}$. Let $T$ be the disjoint union of the $T_{i}$, and $H$ the induced holonomy pseudogroup generated by the $h_{i j}$ (cf. $\S 1.3$.). Given $\omega \in \Omega_{c}^{p+k}(X)$, we can express it as a finite sum $\omega=\sum \omega_{i}$, where the support of $\omega_{i}$ is in $U_{i}$. $\int_{\mathscr{F}} \omega$ will be defined as the class in $\Omega_{c}^{k}(T / H)=\Omega_{c}^{k}(\operatorname{Tr} \mathscr{F})$ of $\Sigma \bar{\omega}_{i}$, where $\bar{\omega}_{i}=\int_{f_{i}} \omega$. The class of $\Sigma \bar{\omega}_{i}$ is independent of the decomposition of $\omega$. Indeed, if $\left\{\lambda_{i}\right\}$ is a partition of 
unity subordinated to $\left\{U_{i}\right\}$, then

$$
\sum_{i} \int_{f_{i}} \omega_{i}=\sum_{i, j} \int_{f_{i}} \lambda_{j} \omega_{i}
$$

is equivalent to

$$
\sum_{i, j} \int_{f_{j}} \lambda_{j} \omega_{i}=\sum_{j} \int_{f_{j}} \lambda_{j} \omega
$$

because if the support of $\alpha$ is in $U_{i} \cap U_{j}$, then

$$
\int_{f_{i}} \alpha=h_{i j}^{*} \int_{f_{j}} \alpha .
$$

It is obvious that this map is continuous and commutes with $d$. One easily shows that it is independent of the choice of the regular covering (by passing to common refinements).

Corollary. The transpose of $\int_{\mathscr{F}}$ gives a linear map

$$
C_{k}(\operatorname{Tr} \mathscr{F}) \rightarrow C_{k+p}(X)
$$

of the space of holonomy invariant $k$-currents in the space of $(p+k)$-currents on $X$. This map commutes with the boundary operator $\delta$.

This is a straightforward generalization of the construction of RuelleSullivan [4] associating to an invariant measure a $p$-current on $X$.

To see an example of a $p$-current on $X$ arising from a holonomy invariant distribution which is not a measure, consider a Reeb foliation like in Example 2.2. Let $L$ be a noncompact leaf, and $\xi$ a vector field along $L$ invariant by holonomy (i.e., projectable with respect to local projections). Let $\omega$ be a 2 -form on $X$, and denote by $\xi . \omega$ its derivative in the direction of $\xi$ (restricted to $L$ ). Then $\int_{L} \xi . \omega$ is finite and defines a 2-current on $X$ which arises from a holonomy invariant distribution of order one.

\subsection{The kernel of $\int_{\mathscr{F}}$}

Following the terminology of [5], a $(p+k)$-form is $\mathscr{F}$-trivial if for any sequence $\xi_{1}, \cdots, \xi_{p+k}$ of vector fields such that $p$ of them are tangent to $\mathscr{F}$, then $\omega\left(\xi_{1}, \cdots, \xi_{p+k}\right)=0$.

Theorem. The kernel of $\int_{\mathscr{F}}$ is the vector subspace generated by $\mathcal{F}$-trivial forms and differential of $\mathcal{F}$-trivial forms.

Proof. We first prove the assertion in the particular case of the foliation given by the natural linear submersion $f: R^{q} \times R^{p} \rightarrow R^{q}$, where $f(x, y)=x$. Any $(p+k)$-form $\omega$ with compact support can be written as $\omega=\alpha+\beta$, 
where $\beta$ is $\mathscr{F}$-trivial, and

$$
\alpha=\sum a_{I} d x^{I} \wedge d y^{1} \wedge \cdots \wedge d y^{p}
$$

where $d x^{I}=d x^{i_{1}} \wedge \cdots \wedge d x^{i_{k}}, 1 \leqslant i_{1}<\cdots<i_{k} \leqslant q$. By assumption, for each $I, \int_{\mathbf{R}^{p}} a_{I}(x, y) d y^{1} \wedge \cdots \wedge d y^{p}=0$. Hence there are smooth $(p-1)$ forms $\gamma_{I}$ on $R^{p}$ depending smoothly on the parameter $x$, such that $d \gamma_{I}=$ $a_{i} d y^{1} \wedge \cdots \wedge d y^{p}$ (cf. [3] where a smooth homotopy operator is constructed). Let $\gamma=(-1)^{k} \sum d x^{i} \wedge \gamma_{I}$. Then $d \gamma=\alpha+\beta^{\prime}$, where $\beta^{\prime}$ is $\mathscr{F}$ trivial. Hence $\omega=d \gamma-\beta^{\prime}+\beta$ where $\gamma, \beta$ and $\beta^{\prime}$ are $\mathscr{F}$-trivial.

We now consider the general case. To construct $\int_{\mathscr{F}}$ we use as before a regular covering of $X$ such that each $f_{i}: U_{i} \rightarrow T_{i}$ is diffeomorphic to a linear projection as above. If $\omega$ is $\mathscr{F}$-trivial, then using a partition of unity we can express it as a finite sum of $\mathscr{F}$-trivial forms $\omega_{i}$ with support in $U_{i}$. Thus it is clear that

$$
\int_{f_{i}} \omega_{i}=0, \int_{f_{i}} d \omega_{i}=0
$$

Conversely, assume that $\int_{\mathscr{F}} \omega=0$. This means that there are $k$-forms $\beta_{j i}$ with compact support in $T_{i}$ such that

$$
\sum_{i} \int_{f_{i}} \omega_{i}=\sum_{i, j} h_{i j}^{*}\left(\beta_{j i}\right)-\beta_{j i}
$$

Hence

$$
\int_{f_{i}} \omega_{i}=\sum_{j} h_{j i}^{*}\left(\beta_{i j}\right)-\beta_{j i}
$$

Let $\alpha_{j i}$ be $(p+k)$-forms with compact support in $U_{i} \cap U_{j}$ such that

$$
\int_{f_{i}} \alpha_{j i}=\beta_{j i}
$$

Note that

$$
\int_{f_{i}} \alpha_{j i}=h_{i j}^{*}\left(\beta_{j i}\right)
$$

hence

$$
\int_{f_{i}} \tilde{\omega}_{i}=0
$$

where

$$
\tilde{\omega}_{i}=\omega_{i}-\sum_{j}\left(\alpha_{i j}-\alpha_{j i}\right)
$$


It follows from the particular case that each $\tilde{\omega}_{i}$ is the sum of a $\mathscr{F}$-trivial form and the differential of a $\mathscr{F}$-trivial form. But this is also true for $\omega$ because $\omega=\Sigma \tilde{\omega}_{i}$.

\subsection{Interpretation of $\Omega_{c}^{0}(\operatorname{tr} \mathscr{F})$}

Let $\Omega_{c}^{r}(\mathscr{F})$ be the vector space of smooth $r$-forms along the leaves (namely, the smooth sections of the $r$ th exterior power of the cotangent bundle of the leaves). The differential $\Omega_{c}^{r}(\mathscr{F}) \rightarrow \Omega_{c}^{r+1}(\mathscr{F})$ along the leaves will be denoted by $d_{0}$. If we denote by $X_{\mathscr{F}}$ the set $X$ which is the union of the leaves of $\mathscr{F}$ and considered as a manifold of dimension $p$, then the identity map $j: X_{\mathscr{F}} \rightarrow X$ is an immersion. $\Omega_{c}^{r}(\mathscr{F})$ is the image in $\Omega^{r}\left(X_{\mathscr{F}}\right)$ by $j^{*}$ of $\Omega_{c}^{r}(X)$, and $d_{0}$ is the restriction to $\Omega_{c}^{r}(\mathscr{F})$ of the differential in $\Omega^{r}\left(X_{\mathscr{F}}\right)$. Let $H^{r}(\mathscr{F})$ be the $r$-th cohomology group of $\Omega_{c}^{*}(\mathscr{F})$. This is (almost by definition) the $r$-th cohomology group of $X$ with value in the sheaf of germs of smooth functions which are constant on the leaves.

Corollary. $\Omega^{0}(\operatorname{Tr} \mathscr{F})$ is canonically isomorphic to $H_{c}^{p}(\mathscr{F})$, where $p=\operatorname{dim} \mathscr{F}$.

Indeed, $\Omega_{c}^{p}(\mathscr{F})$ is just the quotient of $\Omega_{c}^{p}(X)$ by $\mathscr{F}$-trivial forms. Also $j^{*} d \Omega_{c}^{p-1}(X)=d_{0} \Omega_{c}^{p-1}(\mathscr{F})$.

\section{APPLICATIONS TO FOLIATIONS BY MINIMAL LEAVES}

Throughout this section we assume $X$ to be compact and $\mathcal{F}$ oriented. The following theorem is a direct consequence of the preceding section.

4.1. Theorem. A p-form $\omega_{0}$ along $\mathcal{F}$ with compact support is the restriction of a relatively closed form $\omega$ with compact support if $d \int_{\mathscr{F}} \omega_{0}=0$ in $\Omega_{c}^{1}(\operatorname{Tr} \mathscr{F})$.

Proof. Let $\tilde{\omega}$ be a $p$-form with compact support in $X$ such that $\omega_{0}=j^{*} \tilde{\omega}$. As $\int_{\mathscr{F}} \omega_{0}=\int_{\mathscr{F}} \tilde{\omega}$ and $d \int_{\mathscr{F}} \tilde{\omega}=\int_{\mathscr{F}} d \tilde{\omega}=0$, by $\S 3.2$ there is a $p$-form $\alpha \in \Omega_{c}^{p}(X)$ which is $\mathscr{F}$-trivial (i.e., $j^{*} \alpha=0$ ) such that $d \tilde{\omega}-d \alpha$ is $\mathscr{F}$-trivial. Then $\omega=\tilde{\omega}$ $-\alpha$ is relatively closed and $j^{*} \omega=\omega_{0}$.

Corollary. Let $\mathcal{F}$ be an oriented foliation on a compact manifold $X$. Let $g_{0}$ be a smooth riemannian metric along the leaves and let $\omega_{0}$ be the volume form along the leaves defined by $g_{0}$ and the orientation of $\mathcal{F}$. Then there is a riemannian metric $g$ on $X$ inducing $g_{0}$ on the leaves and for which the leaves are minimal submanifolds iff $d \int_{\mathscr{F}} \omega_{0}=0$.

This follows from the above theorem and the theorem of Rummler-Sullivan mentioned in the introduction (cf. [5] and [6]). 
Corollary 2 (Rummler [6]). Suppose that the foliation is a generalized Seifert bundle. Then the metric $g_{0}$ along the leaves extends to a riemannian metric $g$ on $X$ for which all the leaves are minimal iff the volume of each generic leaf $L$ is constant.

Corollary 3. Let $\mathcal{F}$ be a foliation on a compact manifold $X$ given by a closed 1-form $\omega$, and assume that there are at least two $Q$-independent periods of $\omega$. Then any riemannian metric on the leaves can be aproximated in the $C^{\infty}$-topology by a metric which is the restriction to the leaves of a riemannian metric for which the leaves are minimal. If there are two periods whose ratio satisfies a diophantine condition, then any smooth metric on the leaves is the restriction of a metric on $X$ for which the leaves are minimal.

This follows from the considerations in Example 2.1.

Corollary 4. Assume there is no holonomy invariant distribution. Then any riemannian metric $g_{0}$ on the leaves is close in the $C^{\infty}$-topology to a metric which is the restriction of a riemannian metric on $X$ for which the leaves are minimal.

Proof. Let $\omega_{0} \in \Omega^{p}(\mathscr{F})$ be the volume form of $g_{0}$. In any neighborhood of $\omega_{0}$ there is a form $\bar{\omega}_{0}$ such that $\int \bar{\omega}_{0}=0$, because the map $\int_{\mathscr{F}}$ is open and, by assumption, 0 is dense in $\Omega_{c}^{0}(\operatorname{Tr} \mathscr{F})$. Now $\bar{\omega}_{0}$ is the volume form of a riemannian metric on the leaves close to $g_{0}$. So we can apply Corollary 1 .

Remark. More generally, the conclusion of the first part of Corollary 3 is still valid for a transversely $G / H$-homogeneous foliation $\mathcal{F}$ on a compact manifold $X$ with an everywhere dense leaf, assuming $G$ compact connected. In that case, it follows from $\$ 2.1$ that the space of holonomy invariant distributions is isomorphic to $R$. Thus the quotient of $\Omega^{0}(\operatorname{Tr} \mathscr{F})$ by the closure of zero is isomorphic to $\mathbf{R}$, representative for its element being constant functions on $G / H$ (which is compact by assumption). Hence, if $\omega_{0}$ is a volume form on the leaves, then there is a constant $c$ such that $\int \omega_{0}-c$ is adherent to zero. So we can replace $\omega_{0}$ as above by an arbitrary close form $\bar{\omega}_{0}$ such that $\int \bar{\omega}_{0}$ is equivalent to the constant $c$, and hence has zero differential.

Corollary 5. Let $g_{0}$ be a riemannian metric on the leaves of an oriented foliation $\mathcal{F}$ on a compact manifold $X$.A necessary condition for $g_{0}$ to be arbitrarily close to the restriction to the leaves of a metric for which the leaves are minimal is that

$$
\left\langle c, d \int_{\mathscr{F}} \omega_{0}\right\rangle=0
$$

for each holonomy invariant 1-current $c$, where $\omega_{0}$ is the volume form on the leaves defined by $g_{0}$. This condition is also sufficient if $\mathscr{F}$ is transversely oriented and of codimension 1 . 
Proof. The necessity follows from Corollary 1 , and the sufficiency is implied by the following assertion.

Claim. Let $H$ be a pseudogroup of orientation-preserving local diffeomorphisms of a 1-dimensional manifold $T$. Assume that $T$ has a finite number of connected components, and let $f$ be a smooth function with compact support on $T$ such that $\langle c, d f\rangle=0$ for each $H$-invariant 1-current $c$. Then arbitrarily close to $f$ in the $C^{\infty}$-topology, there is a smooth function $g$ such that $d g=0$ in $\Omega_{c}^{1}(T / H)$.

To prove this we can assume that $H$ is irreducible in the following sense: we can order the connected components $T_{i}$ of $T$ so that for each $i$ there is $h_{i} \in H$ with source an open set in $\cup_{j<i} T_{j}$ and target in $T_{i}$. By assumption, there is a sequence $\alpha_{n} \in \Omega_{c}^{1}(T)$ such that $\alpha_{n}$ converges to $d f$ in the $C^{\infty}$-topology and $\alpha_{n}=0$ in $\Omega_{c}^{1}(T / H)$. This implies that $\int_{T} \alpha_{n}=0$, because integration on $T$ gives an invariant current. If the integral of $\alpha_{n}$ on each $T_{i}$ would be zero, then $\alpha_{n}$ would be the differential of a function $f_{n}$ with compact support on $T$, and the sequence $f_{n}$ (modified by suitable constants on the compact components $T_{i}$ ) would converge to $f$.

To achieve this condition, we argue by descending induction on $r$. Assume that $\int_{T_{i}} \alpha_{n}=0$ for each $i>r$. Then one can find a sequence $\alpha_{n}^{\prime}$ such that $\alpha_{n}^{\prime}$ converges to $d f, \alpha_{n}^{\prime}$ is zero in $\Omega_{c}^{1}(T / H)$ and $\int_{T_{i}} \alpha_{n}=0$ for $i>r-1$. Indeed, choose a 1-form $\gamma$ with compact support in the target of $h_{r}$ such that $\int_{T_{r}} \gamma=1$. Then we define

$$
\alpha_{n}^{\prime}=\alpha_{n}-c_{n} \gamma+h_{r}\left(c_{n} \gamma\right)
$$

where $c_{n}=\int_{T_{r}} \alpha_{n}$. Note that $c_{n}$ tends to zero because $\int_{T_{r}} \alpha_{n}$ converges to $\int_{T} d f=0$.

Remark. Corollary 5 implies the following. Let $\mathscr{F}$ be an oriented and transversely oriented foliation of codimension one. Then any metric on the leaves is arbitrarily close to the restriction of a metric on $X$ for which all the leaves are minimal if and only if $\partial C_{1}(\operatorname{Tr} \mathscr{F})=0$, where $\partial: C_{1}(\operatorname{Tr} \mathscr{F}) \rightarrow$ $C_{0}(\operatorname{Tr} \mathscr{F})$ is the dual of $d$.

As an example (besides the one given in Corollary 3), assume that the holonomy pseudogroup of $\mathscr{F}$ is equivalent to the pseudogroup generated by a cocompact subgroup $\Gamma$ of $\mathrm{PSl}_{2}(\mathbf{R})$ acting as usual on $S^{1}$ identified with the boundary of the Poincaré disk $D$. (For instance, $\mathscr{F}$ might be the Anosov foliation associated to the geodesic flow on a compact riemann surface with constant negative curvature). The only $\Gamma$-invariant 1-current on $S^{1}$ are the multiple of the current defined by integration on $S^{1}$. Indeed any 1-current $c$ on $S^{1}$ is the restriction to $S^{1}$ of a harmonic function $f$ on $D$, and if $c$ is $\Gamma$-invariant, then $f$ is also $\Gamma$-invariant, and hence constant because $\Gamma \backslash D$ is compact. So any $\Gamma$-invariant 1 -current has a trivial boundary. 
4.1. Theorem. On the compact manifold $X$ there is a metric such that the leaves of $\mathscr{F}$ are minimal submanifolds iff for a representative $H$ of the holonomy pseudogroup acting on a q-manifold $T$, there is a smooth positive function $f$ with compact support, which is strictly positive on a set intersecting each orbit, and satisfies that $d f=0$ in $\Omega_{c}^{1}(T / H)$.

Before giving the proof of this theorem, we state two corollaries.

Corollary 1. The existence of a riemannian metric for which the leaves are minimal depends only on the holonomy pseudogroup of $\mathscr{F}$.

Corollary 2. If there is a representative $H$ for the holonomy pseudogroup acting on a compact manifold $T$, then there is a metric for which the leaves are minimal.

Indeed we can choose $f \equiv 1$. For instance this is the case if the holonomy pseudogroup is generated by a discrete subgroup of a Lie group acting on a compact manifold. Such an example is given by a foliation defined by $q$ independent closed 1-forms.

Proof of the theorem. First we note that the existence of such an $f$ is independent of the representative for the holonomy pseudogroup.

More precisely, let $H^{\prime}$ be a pseudogroup acting on $T^{\prime}$ which is equivalent to $H$ by an isomorphism $\Phi: H^{\prime} \rightarrow H$. Let $K^{\prime}$ be a compact set intersecting each orbit of $H^{\prime}$. Then there is a positive smooth function $f^{\prime}$ with compact support equivalent to $f$ and which is strictly positive on $K^{\prime}$. To see that, we choose a finite number of $\varphi_{i} \in \Phi, i=1, \cdots, r$, whose domains $U_{i}$ cover $K^{\prime}$ such that $f$ is strictly positive on $\varphi_{i}\left(U_{i}\right)$. One can find a covering of $K$ by compact sets $K_{i} \subset K^{\prime} \cap U_{i}$. Let $\varphi_{j}, r<j \leqslant s$ be elements of $\Phi$ such that the ranges of the $\varphi_{k}, 1 \leqslant k \leqslant s$, cover the support $S$ of $f$. Choose a partition of unity $\lambda_{k}$ subordinated to the covering of $T$ by the ranges of the $\varphi_{k}, 1 \leqslant k \leqslant s$ (and also the complement of $S$ ). We can choose the $\lambda_{i}$ strictly positive on the $\varphi_{i}\left(K_{i}\right)$ for $1 \leqslant i \leqslant r$. Then

$$
f^{\prime}=\sum_{1}^{s} \varphi_{i}^{*}\left(\lambda_{i} f\right)
$$

is the desired function.

Let $\left\{U_{i}\right\}$ be a finite regular covering of $X$ for $\mathscr{F}$ with local projections $f_{i}$ : $U_{i} \rightarrow T_{i}$. We can assume that the $f_{i}$ are diffeomorphic to natural projections $U_{i}=T_{i} \times R^{q} \rightarrow T_{i}$. Let $\left\{V_{i}\right\}$ be a covering of $X$ by compact sets $V_{i}$ contained in $U_{i}$. In each $U_{i}$ we can construct a closed $p$-form $\alpha_{i}$, whose restriction to each plaque $P$ of $U_{i}$ has compact support, is strictly positive on $P \cap V_{i}$, and satisfies $\int_{P} \alpha_{i}=1$. Let $H$ be the holonomy pseudogroup induced on $T=$ union of $T_{i}$. By hypothesis and the preceding considerations, we can find a smooth positive function $f$ with compact support on $T$, which is strictly 
positive on each $K_{i}=f_{i}\left(V_{i}\right)$ and satisfies that $d f=0$ in $\Omega^{1}(T / H)$. Let $g_{i}$ be the restriction of $f$ to $T_{i}$.

Then $\omega=\sum \omega_{i}$. where $\omega_{i}=f_{i}^{*}\left(g_{i}\right) \alpha_{i}$ is a $p$-form on $X$, which is positive on the leaves and whose integral over $\mathscr{F}$ is equivalent to $f$. Then we can apply Corollary 1 of Theorem 4.1.

\subsection{Examples of foliations having no riemannian metric for which the leaves are minimal}

This will be in particular the case for a foliation $\mathcal{F}$ having a positive holonomy invariant measure which is the boundary of an invariant 1-current (cf. [7]). Indeed in this case, for any $p$-form $\omega_{0}$ positive on the leaves, we have

$$
c\left(d \int_{F} \omega_{0}\right)=\partial c\left(\int_{F} \omega_{0}\right)>0 .
$$

For instance in the case of codimension 1 , let $R$ be a Reeb component with boundary $\delta R$; a transversal curve entering $R$ cannot cross the boundary again. The 1-current defined by the integral on positively oriented transversal curves is an invariant current whose boundary is the Dirac measure corresponding to $\delta R$.

In the case of the horocycle flow (cf. Sullivan [6]), one has on the transverse submanifold a positive invariant 2-form which is the exterior differential of an invariant 1 -form (defining an invariant 1-current). This example can be generalized as follows. Let $G$ be a semisimple Lie group acting on a manifold $M$ of dimension $n$ so that the induced action on the space $T_{0}^{*} M$ of nonzero cotangent vectors is transtive. For instance, $G$ might be the conformal group $0(n+1,1)$ acting on the $n$-sphere $S^{n}$ or the linear group $S l(n+1, R)$ acting on $S^{n}$ identified to the rays in $R^{n+1}$.

On $T^{*} M$, one has the canonical 1 -form $\omega$ which is invariant by the differential of any diffeomorphism of $M$, and whose exterior differential $d \omega$ is the canonical symplectic form. Then $(d \omega)^{n}$ is a volume form on $T_{0}^{*} M$, which is the differential of $\omega \wedge(d \omega)^{n-1}$. This form defines a 1-current invariant by the differential of any diffeomorphism, and its boundary is the invariant measure defined by $(d \omega)^{n}$.

Let $\Gamma$ be a discrete subgroup of $G$ such that $\Gamma \backslash G$ is compact. Let $H$ be the subgroup of $G$ leaving a given covector fixed. Then the cosets $g H$ are the leaves of a foliation on $G$ parametrized by the space $T_{0}^{*} M$. This foliation is invariant by the left action of $\Gamma$ on $G$. So we get on $\Gamma \backslash G$ a foliation whose transverse structure is $T_{0}^{*} M$, the holonomy pseudogroup being generated by $\Gamma$. 


\section{References}

[1] M. R. Hermann, Sur le groupe des difféomorphismes du tore, Ann. Inst. Fourier (Grenoble) 23 (1973) 75-86.

[2] J. Plante, Foliations with measure preserving holonomy, Ann. of Math. 102 (1975) 327-361.

[3] G. de Rahm, La théorie des formes différentielles extérieures et l'homologie des variétés différientiables, Rend. Mat. 20 (1961) 105-146.

[4] D. Ruelle \& D. Sullivan, Currents, flows and diffeomorphisms, Topology 14 (1975) 319-327.

[5] H. Rummler, Quelques notions simples en géométrie riemannienne et leurs applicationsn aux feuilletages compacts, Comment. Math. Helv. 54 (1979) 224-239.

[6] __ Kompakte Blätterungen durch Minimalflächen, Habilitationsschrift, Freiburg Universität.

[7] D. Sullivan, A homological characterization of foliations consisting of minimal surfaces, Comment. Math. Helv. 54 (1979) 218-223.

University of Geneva, SWitzerLand 